\title{
A theoretical assessment of heat transfer by ventilation in homogeneous snowpacks
}

\author{
S. J. Bartlett ${ }^{1,2}$ and M. Lehning ${ }^{2}$ \\ Received 15 September 2010; revised 6 January 2011; accepted 10 February 2011; published 7 April 2011.
}

[1] The effects of heat transfer by ventilation in snow are investigated theoretically. We draw together standard analytical results for fluid flow in porous media and apply them to the case of steady flows induced by periodic roughness elements. These solutions are used to estimate the relative magnitude of ventilation heat transfer in snow. We conclude that topography-driven ventilation is unlikely to have a significant impact on the larger scale energy balance of snow-covered regions since the airflow is confined to a shallow penetration depth or just the roughness elements themselves, rather than the bulk snowpack. In particular, for the limiting case of very warm and moisture saturated air flowing over a melting snow cover, we show that latent and sensible heat due to ventilation have about equal contributions and that this contribution is small compared to the overall surface flux as predicted by the Monin-Obukhov theory.

Citation: Bartlett, S. J., and M. Lehning (2011), A theoretical assessment of heat transfer by ventilation in homogeneous snowpacks, Water Resour. Res., 47, W04503, doi:10.1029/2010WR010008.

\section{Introduction}

[2] Snow ventilation is a phenomenon that has long been discussed and analyzed theoretically [Albert, 1996; Clarke and Waddington, 1991; Colbeck, 1989; Cunningham and Waddington, 1993; Waddington et al., 1996] and experimentally [Albert and Hardy, 1995; Sokratov and Sato, 2000]. Simply put, it is the flow of air through the pore space of a snowpack. It is a special case of fluid flow through porous media, a subject which has received considerable attention [e.g., Bear, 1988; Nield and Bejan, 2006]. Porous media flow and heat transfer receive significant interest because of their importance in various geological and industrial contexts, while interest in forced convection through snow has been primarily driven by a need to understand how chemical species in the atmosphere are absorbed by the ice matrix [Cunningham and Waddington, 1993; Waddington et al., 1996]. Over time, such chemical species become locked into polar ice and these chemical records are essential for studying the chemical composition of the atmosphere throughout the Earth's recent history [e.g., Siegenthaler et al., 2005].

[3] For a complete analysis of heat transfer by ventilation in snow we must bring together theoretical results from several different fields. We require the fluid dynamical results of numerical and experimental studies of flow over topography, we require information about the character of the atmospheric surface layer from the field of atmospheric science, we must draw on the theory of fluid flow in porous media, and also the theories of heat transfer by

\footnotetext{
${ }^{1}$ Institute for Complex Systems Simulation, University of Southampton, Southampton, UK.

${ }^{2}$ Snow and Permafrost Research Unit, WSL Institute for Snow and Avalanche Research SLF, Davos, Switzerland.
}

Copyright 2011 by the American Geophysical Union. 0043-1397/11/2010WR010008 forced fluid convection. In this investigation we will aim to assess the impact that snow ventilation might have on the heat flux at a snow surface. Some authors have stressed that ventilation plays a "key role" in heat transfer through snow [Albert and McGilvary, 1992]. Others have concluded that its impact is negligible in the majority of circumstances [Colbeck, 1989]. Based on the results of the present investigation given in section 4.2, we have concluded that it cannot significantly increase the transfer of heat above that of conventional surface exchange since the ventilation flows are too weak and the strongest flows only occur to a small penetration depth.

[4] There are several mechanisms through which ventilation occurs in a snowpack. Shear-driven ventilation can occur within a thin layer of thickness $\sim 5 \mathrm{~mm}$ below the snow surface if topographic features are negligible in height (the snow is only rough at the millimeter scale) [Clifton et al., 2008]. It can also be caused by turbulent fluctuations of air which impose a nonstationary, inhomogeneous pressure distribution upon the snow surface. Long periodic, barometric changes in atmospheric pressure can force air into and out of the snow over the course of synoptic time scale periods [Colbeck, 1989; Cunningham and Waddington, 1993]. Finally, topographic features such as snow dunes can cause a steady, nonuniform pressure distribution to arise inducing stationary ventilation flows within such features. It has been concluded [Clifton et al., 2008] that shear-driven ventilation occurs only within a thin layer beneath the surface and is thus unlikely to induce a large additional heat transfer to the bulk snowpack. Pressure gradients induced by turbulent fluctuations tend to be of a sufficiently high frequency that the corresponding ventilation flows are short lived and rapidly attenuated [Colbeck, 1989]. It is also clear that barometric pressure changes cause the entire body of air within a snowpack to be compressed or expanded over long periods. Over shorter time scales, the heat transfer into and out of the snow will be more heavily influenced by other 
factors. This leaves topography-driven ventilation as the only significant means through which additional heat transfer might occur when steady winds pass over nonuniform snow surfaces. Indeed, other authors have claimed that topography-driven ventilation is the mechanism likely to be significant to heat transfer and chemical uptake [Colbeck, 1989; Cunningham and Waddington, 1993].

[5] The problem of snow ventilation has been investigated theoretically by Clarke and Waddington [1991], Cunningham and Waddington [1993], and Waddington et al. [1996] in the context of the uptake of chemical species. They concluded that topography-driven snow ventilation could have a significant impact on the rate at which certain substances are absorbed by the snowpack. Colbeck [1989] also assessed the problem of snow ventilation, with an approximate estimate of the heat flux due to flows induced by surface features. Again, he concluded that this mode of ventilation could provide an additional means for heat delivery while the other mechanisms, turbulent and barometric, were likely to have a minimal impact. Finally, Albert [1996], Albert and Hardy [1995], and Albert and McGilvary [1992] conducted numerical investigations into the effects of the different means by which heat can be delivered to the snowpack and concluded that topographydriven ventilation should be the most significant.

[6] In this paper we shall provide a compact and tractable analysis of the problem of topography-induced ventilation flows, similar in approach to that by Cunningham and Waddington [1993]. In order to assess the potential influence of ventilation on the overall energy balance of snow covered surfaces, we shall assess the heat flux due to ventilation and compare its magnitude to that of turbulent transfer as predicted by the Monin-Obukhov theory. We shall make several key assumptions in deriving the final result and as a result our conclusions are of a qualitative nature. In the next section we outline some of the basic theory required for an analysis of fluid flow in porous media before deriving analytical solutions to the problem of steady flow through twodimensional snow dunes in section 3 . We shall then apply these results to the problem of heat transfer and compare the approximate magnitudes of ventilation to turbulent heat transfer. Specifically, we shall calculate both sensible and latent heat estimates for the extreme case of warm moist air flowing over a melting snowpack.

\section{Fluid Flow in Porous Media Theory}

[7] We begin by deriving the general equation which describes the propagation of pressure fluctuations through a porous medium. We assume a linear form of Darcy's law for the momentum equation governing the airflow in the snow. Use of this form of the momentum equation as opposed to the Brinkman-Forchheimer extended model [Nield and Bejan, 2006] is justified because most of the airflow occurs sufficiently far from any impermeable boundaries (the soil or ice layer) and the flow is sufficiently slow that inertia effects are not important. Thus we shall use the basic Darcy equation as our starting point [Bear, 1988; Nield and Bejan, 2006]:

$$
\mathbf{u}=-\frac{k_{p}}{\mu} \nabla P
$$

where $\mathbf{u}$ is the volume flux vector of air in the medium, $k_{p}$ is the permeability of the medium (assumed to be isotropic and homogeneous), $P$ is air pressure, and $\mu$ is the dynamic viscosity of air. The permeability $k_{p}$ is a parametrization of the flow resistance of a porous medium, in which tortuosity and porosity effects are embodied. For low speed, laminar flows, the majority of the flow resistance will be caused by frictional drag between the air and the walls of the pore spaces. However for higher flow velocities, inertia will become more important and eventually the flow inside the pores will become turbulent. These are the cases in which Darcy's law is no longer valid and one must use a more sophisticated momentum equation. It is stated by Bear [1988] that "Darcy's law is valid as long as the Reynolds number based on average grain diameter does not exceed some value between 1 and 10". The porous media Reynolds number is defined as $R e_{p}=\mathbf{u} d / \nu$, where $\nu$ is the kinematic viscosity of the fluid. Using a typical snow pore diameter of $d \approx 0.5 \times 10^{-3} \mathrm{~m}$ [Clifton et al., 2008], we are therefore confined to volume fluxes of $\mathbf{u} \leq 0.12 \mathrm{~m} \mathrm{~s}^{-1}$. It would require an extremely strong wind passing over our model roughness elements to produce a volume flux greater than this value. There are no sources or sinks of air in the system (release of vapor through sublimation is neglected) thus the airflow is governed by the continuity equation [Nield and Bejan, 2006]

$$
\phi \frac{\partial \rho}{\partial t}+\nabla \cdot(\rho \mathbf{u})=0,
$$

where $\rho$ is the air density. The porosity $\phi$ is assumed to be homogeneous and stationary (effects of ice structure change due to changes of phase between vapor and ice are assumed to be negligible). We describe the pressure $P$ as being composed of a stationary and homogeneous ambient pressure $P_{0}$ and a time-varying, inhomogeneous pressure fluctuation $p^{\prime}(\mathbf{x}, t)$ of much smaller magnitude,

$$
P(\mathbf{x}, t)=P_{0}+p^{\prime}(\mathbf{x}, t),
$$

where $\mathbf{x}$ is a coordinate vector, $t$ is time, and $P_{0} \gg\left|p^{\prime}\right|$. We also assume that the air obeys the equation of state for an ideal gas,

$$
P=\rho \frac{R T}{M_{a}},
$$

where $R$ is the molar gas constant, $T$ is temperature, and $M_{a}$ is the molar mass of air. Inserting equations (1) and (4) into equation (2), and eliminating $R$ and $M_{a}$ yields

$$
\frac{k_{p}}{\mu \phi} \nabla \cdot\left(\frac{P}{T} \nabla P\right)=\frac{\partial}{\partial t}\left(\frac{P}{T}\right) .
$$

[8] If we assume that the gas is incompressible,

$$
\frac{P}{T}=\rho \frac{R}{M_{a}}=\text { constant }
$$

then $\partial / \partial t(P / T)$ vanishes, as does $\nabla \cdot(P / T)$. Finally, if we make use of the specific form for the pressure (equation (3)), we find that

$$
\nabla^{2} p^{\prime}=0
$$


since $\nabla P_{0}=0$. Equation (7) can be solved straightforwardly given suitable boundary conditions.

\section{Solutions for the Velocity Field}

[9] In this section we will analyze steady ventilation flows caused by topographic features. Dunes and sastrugi are examples of common wind-sculpted structures found in snow-covered regions. If we focus on the mean flow of the wind passing over such features, there will be a nonuniform distribution of dynamic pressure along the surface of the feature. Such pressure gradients drive steady flows within surface roughness elements. We shall derive a simple expression for the ventilation velocity of flows through idealized roughness elements: sinusoidal, two-dimensional dunes. Equation (7) will be used as our governing equation, the solution of which requires two boundary conditions. When a steady flow moves transverse to a solid duneshaped feature there is a high-pressure region upstream of the crest and a lower pressure region on the downstream side of the crest. An exception to this would be when the flow is sufficiently slow as to be described as Stokes or creeping flow, i.e., when $R e \ll 1$. However the atmospheric flows of relevance to this investigation have $R e \gg 1$. In experimental and numerical investigations, (e.g., Cardenas and Wilson [2007]), separation of the momentum boundary layer is clearly visible at the dune crest. The boundary layer reattaches between the trough and the next dune crest. Without detailed values for the exact pressure distribution over our 2-D dune, we will assume that it has the same shape as the dune itself (also sinusoidal with the same wavelength as the dune). This approximation is backed up by some of the sparse experimental studies of flow over sinusoidal dunes [Ho and Gelhar, 1973; Vanoni and Hwang, 1967], which showed that the pressure distribution is approximately the same shape as the topography, but phase shifted with respect to its position (see Figure 1). Our surface pressure distribution, which is one of the boundary conditions, can hence be described by [Colbeck, 1989; Cunningham and Waddington, 1993]

$$
p^{\prime}(x, 0)=P_{d} \sin (K x),
$$

where $K=\frac{2 \pi}{\lambda}$ is the wave number of the roughness elements. Note that the perturbation pressure has taken on the form $p^{\prime}=p^{\prime}(x, z)$ since we are focusing on its stationary component. $P_{d}$ is the magnitude of the pressure perturbation caused by the snow dune and $\lambda$ is the wavelength of the dune. Here we have also invoked a flat surface approximation. This means that we assume the pressure distribution exists along the line $z=0$ rather than the actual snow surface, $z_{s}(x)=H \sin (K x-\psi)$. In this expression $H$ is half the total height of a roughness element and $\psi$ is a phase shift due to the fact that $p^{\prime}$ and $z_{s}$ are not in phase with one another (see Figure 1). A detailed assessment of the validity conditions for this assumption is given by Cunningham and Waddington [1993], and we will give a brief summary here.

[10] For the flat surface approximation to be valid, the aspect ratio of the dune $H / \lambda$ must be small enough that the curvature of the dune does not significantly perturb the paths of the flow from those that would result from the same pressures being imposed upon a plane. Therefore a typical change in height caused by the curvature of the dune must be small compared to the penetration depth of the airflow. This will be most important at the highest curvature points
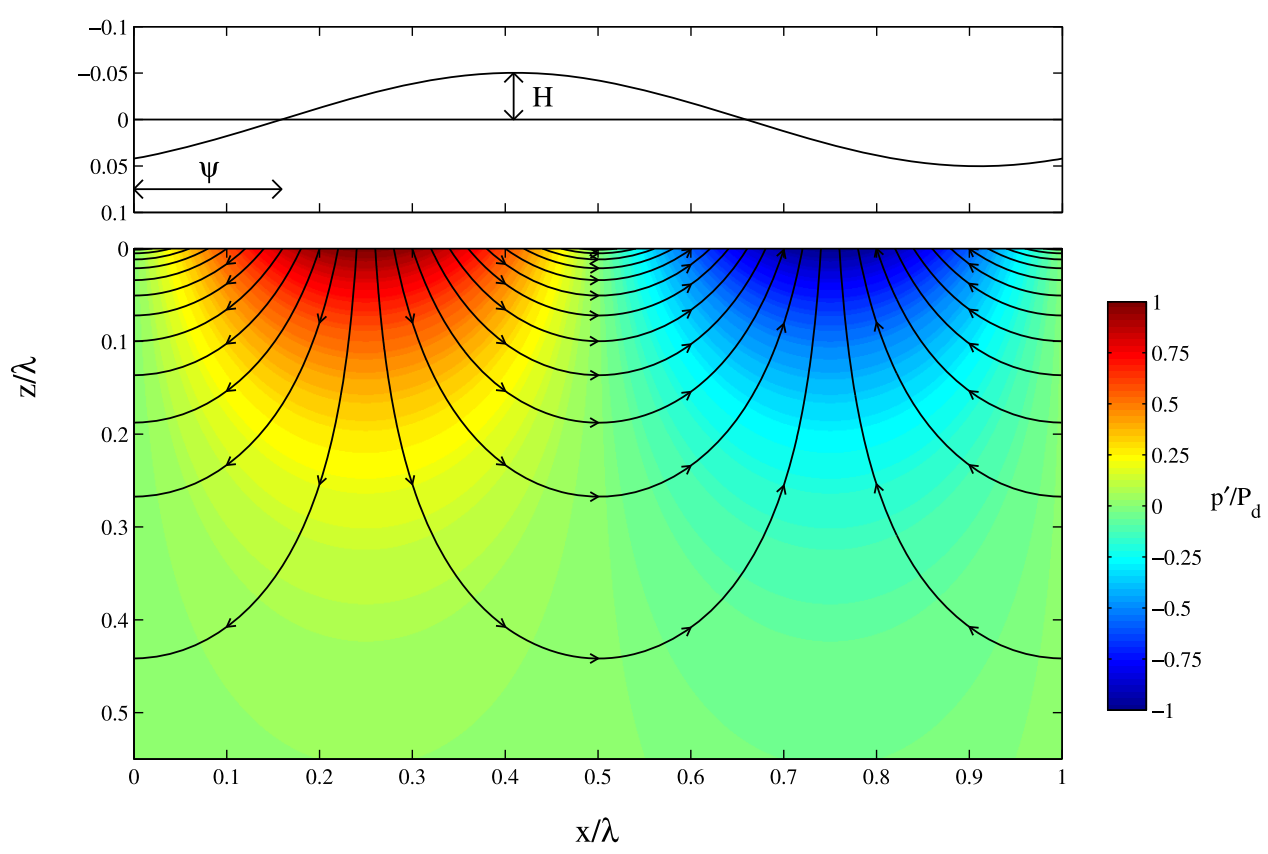

Figure 1. Schematic diagram of our model system. The snow surface consists of sinusoidal roughness elements with an aspect ratio of $H / \lambda=0.05 . \psi$ represents the phase difference between the topography and the dynamic pressure distribution (which has the same functional form as the topography). The position $x$ and depth $z$ are both normalized by the dune wavelength $\lambda$ and apply to both subfigures. The lower figure shows streamlines for the steady flow into the snowpack. Warmer colors represent higher pressures and colder colors represent lower pressures. 
on the dune, i.e., $x=\lambda / 4,3 \lambda / 4, \ldots$. The condition can be expressed by [Cunningham and Waddington, 1993]

$$
\Delta z \ll \lambda / 2 \pi
$$

where the height change caused by moving a horizontal distance $\lambda / 2 \pi$ is given by

$$
\Delta z=\left|H-H \sin \left(\frac{2 \pi}{\lambda} x \pm 1\right)\right|
$$

and hence at $x=\lambda / 4$ the inequality (9) becomes

$$
\begin{aligned}
2 \pi \frac{H}{\lambda}[1-\cos (1)] & \ll 1, \\
\frac{H}{\lambda} & \ll 0.35 .
\end{aligned}
$$

[11] To put this condition into perspective, a dune with an aspect ratio of $H / \lambda=0.05$ is shown in Figure 1. Clearly the flat surface approximation prevents us from interpreting our solution quantitatively for many potential dunes of interest, however periodic roughness elements of low aspect ratio do exist in Antarctica in the form of so-called megadunes [see, e.g., Fahnestock et al., 2000].

[12] The second boundary condition for the solution of the governing equation must come from the lower boundary of the domain. The pertinent condition is that the lower boundary is impermeable, therefore the pressure gradient, because it is proportional to the flow velocity, must vanish there,

$$
\frac{\partial p^{\prime}(x, L)}{\partial z}=0
$$

where $L$ is the depth of the snowpack. The solution to our governing equation, given the boundary conditions, is therefore

$$
p^{\prime}(x, z)=P_{d} \sin K x(\cosh K z-\tanh K L \sinh K z) .
$$

[13] We are now in a position to calculate the air velocity within the snowpack. Using Darcy's law (equation (1)), the air flux is given by

$$
\left\{\begin{array}{l}
u_{x} \\
u_{z}
\end{array}\right\}=\frac{K k_{p} P_{d}}{\mu}\left\{\begin{array}{l}
\cos K x(\tanh K L \sinh K z-\cosh K z) \\
\sin K x(\tanh K L \cosh K z-\sinh K z)
\end{array}\right\} .
$$

[14] We have reliable estimates for all the parameters in this expression except for the magnitude of the pressure perturbation caused by the dune $P_{d}$. At this point, we again return to the work of Cunningham and Waddington [1993] who calculated an approximate value for this parameter. They assumed, as Colbeck [1989] did, that the pressure magnitude is of the form

$$
P_{d}=C \rho \frac{H}{\lambda} U_{b}^{2}
$$

where $C$ must be determined from experiment, $\rho$ is the fluid density, and $U_{b}$ is the free stream velocity away from the momentum boundary layer of the flow over the dune. The form of equation (15) is based on the fact that the dynamic pressure distribution over a cylinder is also sinusoidal and is proportional to $\rho U_{\infty}^{2}$ [Tritton, 1988]. A similar result was found for sand dunes by Vanoni and Hwang [1967]. Using the pressure measurements of Cunningham and Waddington [1993], we shall choose a value of $C=4$ because that is approximately the value they calculated for shapes most similar to our 2-D dunes. They also found that the above analysis breaks down for roughness Reynolds numbers $R e_{r}$ $<70$, because the influence of the shear stress from the snow surface is such that the flow begins to transition to Stokes flow. So if we make use of equation (15), we can only apply the results to situations where the flow is in the aerodynamically rough regime since if the flow was laminar, the magnitude of the dynamic pressure perturbation would instead depend linearly on the freestream velocity $U_{b}$. It should also be noted that Cunningham and Waddington [1993] concluded that $C$ is likely to depend nontrivially on the form of the roughness elements, but to establish such a relationship would require its own dedicated investigation. Nevertheless, we can proceed with estimates for values of the ventilation velocity within dune-like features as long as the parameters conform to the above conditions. We can now assess the form of the flow within the snowpack using equation (14). We can see its solenoidal nature in Figure 1. We also observe that due to the exponential decay of the air velocity with depth and the spacing of the streamlines, the strongest airflow is confined to a thin layer just beneath the snow surface.

\section{Heat Transfer Estimates}

\subsection{Heat Flux Relation}

[15] The total heat flux into the snowpack is a sum of contributions from several different effects [Zhekamukhov and Zhekamukhova, 2002]:

$$
q=-k_{m} \nabla T-L_{w / i} D_{v} \nabla \rho_{v}+\mathbf{u} \rho c_{p} \Delta T+\mathbf{u} L_{w / i} \Delta \rho_{v},
$$

where $k_{m}$ is the weighted thermal conductivity of the snowair system, $L_{w / i}$ is the latent heat of sublimation (w) or vaporization $(i), D_{v}$ is the effective mass diffusivity of water vapor, $\Delta T$ is the temperature difference between the surface air and the interior of the snowpack, $\Delta \rho_{v}$ is the water vapor density difference between the surface air and the interior of the snowpack, $\rho$ is the air density, $c_{p}$ is the specific heat capacity of air at constant pressure, and $\mathbf{u}$ is the volume flux of air (Darcy velocity). The first term of equation (16) represents heat flow by diffusion, the second term represents vapor flow by diffusion (latent heat transfer), the third term accounts for sensible heat transfer by forced convection (ventilation), and the last term represents latent heat transfer by forced convection. The numerical investigations of Albert and McGilvary [1992] concluded that the transport of vapor and the associated latent heat exchange had a significantly lower impact on heat transfer than the transport of heat by air. However, we shall investigate the contribution from latent heat alongside that of sensible heat.

[16] The aim of this investigation was to establish how much extra heat (if any) is delivered to the snowpack through the process of ventilation. Clearly if we wished to obtain an exact expression for the heat flux we would have 
to solve the coupled governing equations for the vapor density and temperature fields. This would have to be performed numerically as was done by Albert [1996]. However, we are interested specifically in the additional contribution due to ventilation processes and we seek simple estimates for the magnitude of such effects. We can approximate the contribution to the sensible and latent heat flux from ventilation by neglecting the diffusion terms of equation (16):

$$
q_{v}=\mathbf{u} \rho c_{p} \Delta T+\mathbf{u} L_{w / i} \Delta \rho_{v} .
$$

[17] This equation simply estimates the heat content (sensible and latent) of a packet of impinging air, assumes it comes into equilibrium with the snow by the time it leaves the snowpack, and uses its velocity as a measure of the rate at which it can deliver its thermal energy. It is important now to distinguish between the key temperatures which will affect heat flux into a snowpack. In addition to the sensible heat flux, these temperatures will also be used to calculate the relevant vapor densities for the latent heat flux.

[18] Clearly the air away from the thermal boundary layer will remain at the ambient freestream temperature $T_{\infty}$. As we move down toward the snow surface, through the thermal boundary layer, the temperature will most likely remain fairly constant through the outer region due to enhanced turbulent mixing, until finally shifting toward the snow surface temperature $T_{s}$ in the inner region. The temperature will continue to change as we move into the snowpack where the thermal boundary layer will extend through a thin layer beneath the snow surface [Ochoa-Tapia and Whitaker, 1997; Vafai and Thiyagaraja, 1987]. Once away from the surface thermal boundary layer the temperature should settle to its "inner" temperature $T_{i}$, the last of our key temperatures. Of course there will be a final thermal boundary layer at the bottom of the snowpack where the temperature changes from $T_{i}$ to the temperature of the solid layer below, but that is sufficiently far from the surface that it does not affect our analyses. If we wish to invoke the approximate formula for the heat flux (equation (17)), we must first possess a knowledge of the internal temperature of the snow $T_{i}$ and the temperature of the impinging air. On average, the temperature of this air should be close to $T_{S}$ however it is also possible that some encroaching eddies could be significantly warmer. It is important to note that our key temperatures are time averages over a turbulent time scale of the atmospheric surface layer (minutes) and do not apply to particular turbulent eddies. In any case, it is this air (at a temperature equal to $T_{S}$ on average) which will enter the snow and undergo ventilation. We then have $\Delta T=T_{s}-T_{i}$. The temperature relevant to ventilation heat transfer is not the freestream air temperature $T_{\infty}$.

\subsection{2-D Steady Flow Through Shallow Dunes}

[19] We shall now attempt to find some characteristic values for the heat flux caused by topography-driven ventilation in snow. Without detailed data sets containing spatial and temporal pressure distributions, we are limited to making qualitative estimates. We will make use of the analysis of section 3 for the velocity field and of section 4.1 for the heat flux relation. We first need to establish the total flux of air into the snow. This is found by integrating the vertical component of the volume flux at the surface over the high pressure portion of the snow dune [Cunningham and Waddington, 1993]:

$$
f=\frac{2}{\lambda} \int_{0}^{1} \int_{0}^{\lambda / 2} u_{z}(x, 0) d x d y=\frac{4 k_{p} P_{d}}{\lambda \mu} \tanh K L .
$$

[20] An equal volume will leave the snow dune over the low-pressure portion at the same rate since the airflow is solenoidal. The tanh $K L$ factor embodies flow path perturbations caused by the presence of an impermeable floor. However, the additional drag induced by such a boundary was not incorporated in our momentum equation. Furthermore, this factor tends to 1 as the depth of the snowpack becomes greater than the wavelength of the roughness elements, therefore it shall be omitted from this point on. The heat content of a unit volume of air is given by

$$
g=\rho c_{p} \Delta T+L_{w / i} \Delta \rho_{v}
$$

and consequently the rate at which heat is brought into the snowpack due to ventilation is found by multiplying equations (18) and (19):

$$
q_{v} \approx \frac{4 k_{p} P_{d}}{\lambda \mu}\left(\rho c_{p} \Delta T+L_{w / i} \Delta \rho_{v}\right) .
$$

[21] This equation is not exact because we see from Figure 1 that the air which enters the snow near $x=0$ and $x=\lambda / 2$ spends very little time in the snowpack because it has a relatively high velocity and short flow path. Therefore it is likely that such air would not spend long enough in the snowpack to come into thermal equilibrium with it and the use of equation (20) might overestimate the heat flux. However, we shall see below that despite the heat flux being slightly augmented in this respect, the implication of our main result remains unaltered. We can now introduce the approximation for the pressure perturbation $P_{d}$ (equation (15)) as given by Cunningham and Waddington [1993],

$$
q_{v} \approx \frac{16 k_{p} H U_{b}^{2} \rho}{\lambda^{2} \mu}\left(\rho c_{p} \Delta T+L_{w / i} \Delta \rho_{v}\right) .
$$

[22] Note that this result only applies when $H / \lambda \ll 0.35$ and when the wind flow is in the aerodynamically rough regime. The total turbulent heat flux is given by [Brutsaert, 2005]

$$
\begin{aligned}
H+E L_{w / i} \approx & \frac{k_{c}^{2} U_{\infty}}{\left[\ln \left(\frac{z-d}{z_{0}}\right)\right]^{2}}\left\{\rho c_{p}\left(T_{\infty}-T_{s}\right)\right. \\
& \left.+L_{w / i}\left[\rho_{v}\left(T_{\infty}\right)-\rho_{v}\left(T_{s}\right)\right]\right\},
\end{aligned}
$$

where $U_{\infty}$ is the freestream wind velocity. We shall assume we are dealing with a snow-covered region where the wind has sculpted sinusoidal dunes with an aspect ratio of $H / \lambda=0.05$, which are transverse to the prevailing wind and which have a wavelength of $\lambda=10 \mathrm{~m}$. The magnitudes of ventilation and turbulent heat fluxes can now be compared. We perform this calculation for the two contributions 
(sensible and latent heat) separately. The ratio of ventilation to turbulent-driven sensible heat flux is given by

$$
\frac{q_{v_{s}}}{H} \approx \frac{16 k_{p} H \rho^{2} c_{p}\left(T_{s}-T_{i}\right)}{\lambda^{2} \mu} \frac{\left[\ln \left(\frac{z-d}{z_{0}}\right)\right]^{2}}{k_{c}^{2} \rho c_{p}\left(T_{\infty}-T_{s}\right)} \frac{U_{b}^{2}}{U_{\infty}} \approx \alpha \frac{U_{b}^{2}}{U_{\infty}}
$$

and for latent heat by

$$
\begin{aligned}
\frac{q_{v_{l}}}{E L_{w / i}} & \approx \frac{16 k_{p} H \rho L_{w / i}\left[\rho_{v}\left(T_{s}\right)-\rho_{v}\left(T_{i}\right)\right]}{\lambda^{2} \mu} \frac{\left[\ln \left(\frac{z-d}{z_{0}}\right)\right]^{2}}{k_{c}^{2} L_{w / i}\left[\rho_{v}\left(T_{\infty}\right)-\rho_{v}\left(T_{s}\right)\right]} \frac{U_{b}^{2}}{U_{\infty}} \\
& \approx \beta \frac{U_{b}^{2}}{U_{\infty}}
\end{aligned}
$$

where $\alpha=1.2 \times 10^{-2} \mathrm{~m} \mathrm{~s}^{-1}$ and $\beta=1.5 \times 10^{-2} \mathrm{~m} \mathrm{~s}^{-1}$. Note that this result applies when the snowpack depth is large compared to the wavelength of the snow dunes. In the case of shallower snowpacks for which the depth is of similar magnitude to the horizontal length scale of the roughness elements, $\alpha$ and $\beta$ will be reduced. Their magnitudes will decay rapidly as the snow depth becomes very small, i.e., as $\lambda / L$ becomes large. We can only estimate this effect since we would need to use a momentum equation which includes inertia terms to gain a complete understanding of the effect of the impermeable floor. However, we can be sure that the ventilation flows will only be reduced by the additional drag. We have assumed that the permeability of the snow is $k_{p}=3 \times 10^{-9} \mathrm{~m}^{2}$ [Sommerfeld and Rocchio, 1993], that the density of the air is $\rho=1.3 \mathrm{~kg} \mathrm{~m}^{-3}$, that the viscosity of the air is $\mu=1.7 \times 10^{-5} \mathrm{~kg} \mathrm{~m} \mathrm{~s}^{-1}$, the temperature differences are $\Delta T=T_{s}-T_{i}=5^{\circ} \mathrm{K}$ and $T_{\infty}-T_{s}=5^{\circ} \mathrm{K}$. We have thus treated the situation of warm, moist air $\left(T_{\infty}=\right.$ $\left.10^{\circ} \mathrm{C}\right)$ passing over a melting snow cover $\left(T_{i}=0^{\circ} \mathrm{C}\right)$. Calculation of the vapor densities was performed using the following semiempirical equation [Lehning et al., 2002]:

$$
\rho_{v}^{w / i}(T)=p_{t} e^{\frac{L_{w / i}\left(T-T_{t}\right)}{R_{v} T_{T} T}},
$$

where $\rho_{v}^{w / i}$ is the saturation vapor pressure over water or ice, $p_{t}$ is the triple point pressure, $T_{t}$ is the triple point temperature, and $R_{v}$ is the water vapor gas constant. This equation is sufficiently accurate given our semiquantitative estimates and our temperatures around the melting snow.

[23] We know that $U_{b} / U_{\infty}<1$, therefore even if $U_{b} \sim 10$ $\mathrm{m} \mathrm{s}^{-1}$, then $q_{v_{s}} / H \approx q_{v_{i}} / E L_{w / i} \sim 10^{-2}$. This implies that ventilation is likely to have a minor impact on heat delivery when we compare it to the conventional prediction of turbulent surface exchange. Since several of the parameters embedded within the constants $\alpha$ and $\beta$ have considerable variability depending on local climatic conditions, it is clear that under certain circumstances topography-driven ventilation may be capable of influencing total heat transport to the snowpack more than our estimation above. For example, if the permeability was relatively high (e.g., $10 \times$ $\left.10^{-3} \mathrm{~m}^{2}\right), T_{s}-T_{i}$ was also large and $T_{\infty}-T_{s}$ was small, $\alpha \rightarrow 10^{-1}$. Under very high winds, if the velocities $U_{b}$ and $U_{\infty}$ were large and the wavelength of the roughness elements was small, it is possible that ventilation-driven heat flux contributions may increase by one order of magnitude. However, it should also be noted that equations (23) and (24) may overestimate the ventilation heat flux in certain situations. Inertial drag was not included in the governing momentum equation, therefore stronger flows may experience greater flow resistance than our analysis incorporates, thus reducing the ventilation volume flux. In addition, snowpacks whose depth are small compared to the wavelength of the roughness elements will exert additional drag on ventilation flows due to the presence of the impermeable floor. And given that we used typical values in reaching equation (23), it seems unlikely that ventilation will consistently have a large impact on heat transfer since its strength is approximately two orders of magnitude lower than that of turbulent transport at the snow surface.

[24] It is possible that surface features with larger aspect ratios may induce stronger ventilation flows. However, as the aspect ratio of the structure increases it is unlikely that the strength of the ventilation flows would increase considerably faster than linearly (see equations (14) and (15)). Furthermore, the magnitude of such flows would still decay exponentially with depth and thus would likely be confined to the features themselves [Clifton et al., 2008]. Thus there is unlikely to be considerable extra heat transported to the bulk of the snowpack.

\section{Discussion and Conclusions}

[25] Based on previous analytical results (not reported here) and in concurrence with other authors [Colbeck, 1989; Cunningham and Waddington, 1993], we have concluded that topography-driven ventilation is likely to be the most significant of all the mechanisms by which ventilation might deliver additional heat to a snowpack. Shear-driven ventilation has been shown to only occur to a shallow depth [Clifton et al., 2008], barometric windpumping has too long a period, and turbulent fluctuations are too high frequency and intermittent. Therefore we have restricted ourselves to topography-driven ventilation here. We have produced qualitative evidence that topography-driven ventilation should have a minimal impact on heat transfer under typical conditions. This does not exclude the possibility that in special cases it could provide an additional contribution to the heat flux at certain locations. Roughness elements with larger aspect ratios (e.g., large magnitude snow dunes) may induce stronger ventilation flows but it is improbable that they would penetrate into the bulk snowpack. Furthermore, snowpacks which have depths smaller than the wavelength of their surface features will experience an even smaller additional heat flux due to ventilation. Finally it should be noted that the Monin-Obukhov theory accommodates the small additional effect of ventilation. Seen from the atmosphere, the presence of ventilation would result in a somewhat higher local roughness length than one could (aerodynamically) measure for the same surface if it were impermeable. However the heat (as well as other scalars and momentum) is still subject to the turbulent transfer in the atmospheric surface layer and therefore captured by the Monin-Obukhov theory. It would be a useful and instructive extension to the work presented here to perform an experimental investigation of topography-driven ventilation and its associated heat transfer. 
[26] Acknowledgments. This work has partially been funded by the Swiss National Science Foundation. We thank Andy Clifton, Costantino Manes, Hendrik Huwald, and Chad Higgins for stimulating discussions. The comments of the reviewers helped to improve the clarity of the presentation. Most of all we want to thank John Selker for motivating this work, for supporting the paper generation, and for his help with the final form.

\section{References}

Albert, M. R. (1996), Modeling heat, mass and species transport in polar firn, Ann. Glaciol., 23, 138-143.

Albert, M. R., and J. P. Hardy (1995) Ventilation experiments in a seasonal snow cover, in Biogeochemistry of Seasonally Snow-Covered Catchments, edited by K. A. Tonnessen et al., pp. 41-49, Int. Assoc. of Hydrol. Sci., Wallingford, UK.

Albert, M. R., and W. R. McGilvary (1992), Thermal effects due to air flow and vapor transport in dry snow, J. Glaciol., 38, 273-281.

Bear, J. (1988), Dynamics of Fluids in Porous Media, Dover, New York.

Bejan, A. (1995), Convection Heat Transfer, John Wiley, New York.

Brutsaert, W. (2005), Hydrology: An Introduction, Cambridge University Press, Cambridge.

Cardenas, M. B., and J. L. Wilson (2007), Hydrodynamics of coupled flow above and below a sediment-water interface with triangular bedforms, Adv. Water Resour., 30, 301-313.

Clarke, G. K. C., and E. D. Waddington (1991), A three-dimensional theory of wind pumping, J. Glaciol., 37(125), 89-96.

Clifton, A., C. Manes, J.-D. Rüedi, M. Guala, and M. Lehning (2008), On shear-driven ventilation of snow, Boundary-Layer Meteorol., 126, 249-261.

Colbeck, S. C. (1989), Air movement in snow due to windpumping, J. Glaciol., 35(120), 209-213.

Cunningham, J., and E. D. Waddington (1993), Air flow and dry deposition of non-sea salt sulfate in polar firn: Paleoclimatic implications, Atmos. Environ., 27A(17/18), 2943-2956.

Fahnestock, M. A., T. A. Scambos, C. A. Shuman, R. J. Arthern, D. P. Winebrenner, and R. Kwok (2000), Snow megadune fields on the East Antarctic Plateau: Extreme atmosphere-ice interaction, Geophys. Res. Lett., 27, 3719-3722, doi:10.1029/1999GL011248.

Ho, R. T., and L. W. Gelhar (1973), Turbulent flow with wavy permeable boundaries, J. Fluid Mech., 58, 403-414.
Lehning, M., P. Bartelt, B. Brown, and C. Fierz (2002), A physical SNOWPACK model for the Swiss avalanche warning: Part III: Meteorological forcing, thin layer formation and evaluation, Cold Reg. Sci. Technol., $35(3), 169-184$.

Nield, D. A., and A. Bejan (2006), Convection in Porous Media, Springer, New York.

Ochoa-Tapia, J. A., and S. Whitaker (1997), Heat transfer at the boundary between a porous medium and a homogeneous fluid, Int. J. Heat Mass Transfer, 40(11), 2691-2707.

Siegenthaler, U., et al. (2005), Stable carbon cycle-climate relationship during the late Pleistocene, Science, 310(5752), 1313-1317.

Sokratov, S. A., and A. Sato (2000), Wind propagation to snow observed in laboratory, Ann. Glaciol., 31, 427-433.

Sommerfeld, R. A., and J. E. Rocchio (1993), Permeability measurements on new and equitemperature snow, Water Resour. Res., 29, 2485-2490, doi:10.1029/93WR01071.

Tritton, D. J. (1988), Physical Fluid Dynamics, Oxford University Press, Oxford.

Vafai, K., and R. Thiyagaraja (1987), Analysis of flow and heat transfer at the interface region of a porous medium, Int. J. Heat Mass Transfer, $30(7), 1391-1405$.

Vafai, K., and C. L. Tien (1981), Boundary and inertia effects on flow and heat transfer in porous media, Int. J. Heat Mass Transfer, 24, $195-203$.

Vanoni, V. A., and L.-S. Hwang (1967), Relation between bed forms and friction in streams, J. Hydr. Div. Am. Soc. Civ. Eng, 93, 121-144.

Waddington, E. D., J. Cunningham, and S. L. Harder (1996), The effects of snow ventilation on chemical concentrations, in Chemical Exchange Between the Atmosphere and Polar Snow, edited by E. W. Wolff and R. C. Bales, Vol. 43 of NATO ASI Series, pp. 403-452, Springer, Berlin.

Zhekamukhov, M. K., and I. M. Zhekamukhova (2002), On convective instability of air in the snow cover, J. Eng. Phys. Thermophys., 75(4), $849-858$

S. J. Bartlett, Institute for Complex Systems Simulation, University of Southampton, Electronics and Computer Science Building 16, Southampton SO17 1BJ, UK. (S.J.Bartlett@soton.ac.uk)

M. Lehning, Snow and Permafrost Research Unit, WSL Institute for Snow and Avalanche Research SLF, Davos, Switzerland, CH-7260. 